\title{
CUSPS OF THE PROJECTIVE GAUSS MAP
}

\author{
CLINT MCCRORY \& THEODORE SHIFRIN
}

\section{Introduction}

Let $M$ be a smooth algebraic surface in complex projective space $\mathbf{P}^{3}$. Let $\gamma$ : $M \rightarrow \mathbf{P}^{3 *}$ be its Gauss map and $\Pi \subset M$ the parabolic curve (the locus of singular points of $\gamma$ ). The image of $\gamma$ is the classical dual surface of $M$. We call the critical points of $\gamma \mid \Pi$ the cusps of the Gauss map $\gamma$. A (nonplanar) cusp $P$ is characterized by the fact that the (unique) asymptotic direction at $P$ is tangent to $\Pi$. We have the following local interpretation of the cusps of the Gauss map, which we prove for smooth real surfaces as well.

(0.1) Theorem. For a generic smooth surface, the cusps of the Gauss map are precisely the parabolic points which are the limits of flex points of asymptotic curves.

This theorem is the complex projective version of a theorem for real smooth surfaces due to Arnold [3], Banchoff, Gaffney and McCrory [6], Kergosien and Thom [13], Landis [17], and Platonova [21]. A discussion of this and related results for real surfaces, from the viewpoint of singularity theory, is contained in a recent survey article of Arnold $[4, \S 15]$. Theorem $(0.1)$ was originally observed by Salmon [22, $§ 588 \mathrm{ff}$.], using global techniques.

The novelty of our approach lies largely in the application of techniques of projective differential geometry in the spirit of Cartan [9], rather than those exclusively of singularity theory. We begin in $\$ 1$ by developing the necessary tools, discussing in particular the projective Gauss mapping and its relation to the projective second fundamental form. As in the classical differential geometry of surfaces in $\mathbf{R}^{3}$, the notions of parabolic points and asymptotic curves prove essential. In order to prove Theorem (0.1), we assume $M$ has no planar points and let $\tilde{M} \subset \mathbf{P}(T M)$ be given by $\tilde{M}=\left\{v \in \mathbf{P}\left(T_{x} M\right): v\right.$ is an asymptotic direction at $x\}$. Then $\tilde{M} \rightarrow M$ is a branched double cover with branch locus the parabolic curve. Lie considered such a construction in studying implicit

Received September 14, 1983. The first author was supported in part by NSF grant MCS-8102759, and the second in part by University of Georgia Faculty Research Grant. 
ordinary differential equations (cf. [2, pp. 14 ff.]). It turns out that we should view $\tilde{M}$ as a submanifold of $\mathbf{P}\left(T^{*} M\right)$, which is naturally isomorphic to $\mathbf{P}(T M)$, in order to exploit the contact structure arising from the symplectic structure on $T^{*} M$. Then the Gaussian cusps and asymptotic flex points have natural geometric interpretations, and the theorem follows. Indeed, we prove a more precise statement: the curve of asymptotic flex points is tangent to the parabolic curve at the points of intersection of the two curves.

In $\S 2$ we pass from the local to the global. We begin by combining the theory of the projective second fundamental form with a Chern class computation to prove a result of classical enumerative geometry (cf. [22], [20]):

(0.2) Theorem. A generic surface of degree d in $\mathbf{P}^{3}$ has $2 d(d-2)(11 d-24)$ Gaussian cusps.

For example, a quadric has none (having, in particular, no parabolic points at all); a cubic has 54 cusps, as the parabolic curve meets each of the twenty-seven lines on the surface (which comprise the asymptotic flex curve) precisely twice. (Kulikov gives a beautiful modern singularity-theoretic derivation of (0.2) in [16] (cf. also [16a]), arriving at several other formulas of interest as well.)

Indeed, after proving $(0.2)$ we proceed to discuss the example of the general cubic surface quite carefully from a classical standpoint. It is natural, then, to consider the Fermat cubic $x_{0}^{3}+x_{1}^{3}+x_{2}^{3}+x_{3}^{3}=0$. While it fails to meet our genericity assumptions, we are nevertheless able to carry out our geometric constructions with the asymptotic double cover and obtain the same count. In this case, it is interesting to note that the parabolic curve is reduced, but reducible, with 18 nodes, which are of necessity planar points. $\tilde{M}$ is smooth and blows up each of the nodes.

When we pass to the Fermat quartic, however, the situation is more subtle-the parabolic curve becomes unreduced and $\tilde{M}$ singular. Nevertheless, the count in $(0.2)$ goes through. In fact, understanding this more involved example leads us to the more general

(0.3) Theorem. Let $M \subset \mathbf{P}^{3}$ be a smooth surface which is neither a plane nor a quadric. Then the (homological) intersection number of the parabolic curve and the curve of asymptotic flexes is precisely $4 d(d-2)(11 d-24)$.

The proof involves an intrinsic reinterpretation of our computations with $\tilde{M}$ in $\$ 1$ and a straightforward continuity argument. In this regard, we remark that while our proof involves computing the intersection number of three surfaces in $\mathbf{P}\left(T^{*} M\right)$, Salmon employs elimination theory to do an analogous computation in $\mathbf{P}^{3}$ itself.

The precise generic properties which we need to require of the surface are that $\Pi$ be smooth and that the cusp points be nondegenerate points of 
tangency of the asymptotic line field with the parabolic curve. These properties are equivalent to the statement that the locus of local singularities of the dual surface is a cuspidal edge with isolated swallowtail points. Using techniques of singularity theory developed by Mather [19], we prove in $\$ 3$ that, for each degree $d>1$, surfaces with these properties form a nonempty Zariski open subset of the space of all smooth surfaces of degree $d$. This is a refinement of a theorem of Bruce [8], and we use his work in an essential manner. We also include, for completeness, the appropriate genericity statement for the real, smooth case. We note that our genericity hypothesis is weaker, and therefore more easily computable, than the hypothesis arising in [6, Theorem 7.6].

A pleasant aspect of these investigations is the interplay among several closely related geometric viewpoints: the dual surface, contact with planes or lines, versal unfolding of tangent hyperplane sections, integral curves of the asymptotic line field. Much of this geometry was perceived by Klein in his study of cubic surfaces $[15, \S 14]$.

We wish to thank Robert Varley for several valuable conversations, and for his continued interest.

\section{Local theory}

We are interested here in the local projective differential geometry of surfaces in three-space; our results in this section are equally valid in the real and complex cases, but we will write just $\mathbf{C}$ (when necessary). We begin by relating the projective Gauss mapping of a surface $M \subset \mathbf{P}^{3}$ and the projective second fundamental form of $M$.

Let $\pi: \mathbf{C}^{4}-\{0\} \rightarrow \mathbf{P}^{3}$ be the canonical projection. We say that a basis $Z_{0}, Z_{1}, Z_{2}, Z_{3}$ for $\mathbf{C}^{4}$ is a frame at $z \in \mathbf{P}^{3}$ if $\pi\left(Z_{0}\right)=z$. A frame on an open set $U \subset \mathbf{P}^{3}$ is therefore a holomorphic (or smooth) map $U \rightarrow G l(4)$ such that $\pi\left(Z_{0}(z)\right)=z . Z_{0}: U \rightarrow \mathbf{C}^{4}$ is in this way a section of the tautological line bundle $\vartheta_{U}(-1)$. Now, given a surface $M \subset \mathbf{P}^{3}$, it is important to have the notion of frames adapted to $M$. At $z \in M$, we require $\pi\left(Z_{0}\right)=z$, as before, and also that $\pi\left(Z_{0} \wedge Z_{1} \wedge Z_{2}\right)$ is the $\mathbf{P}^{2} \subset \mathbf{P}^{3}$ which is tangent to $M$ at $z$. To distinguish the projective tangent space from the abstract tangent space, we shall use the notation $\tilde{T}_{z} M$ for the former, using it to represent both the $\mathbf{C}^{3} \subset \mathbf{C}^{4}$ and the corresponding $\mathbf{P}^{2} \subset \mathbf{P}^{3}$.

Given a (local) adapted frame field $Z_{0}, Z_{1}, Z_{2}, Z_{3}$ on $M$, define 1-forms $\omega_{i}^{j}$, $0 \leqslant i, j \leqslant 3$, on $M$ as follows:

$$
d Z_{i}=\sum_{j=0}^{3} \omega_{i}^{j} Z_{j}
$$


we shall write $\omega_{0}^{1}=\omega^{1}$ and $\omega_{0}^{2}=\omega^{2}$, and these form a (local) basis for the 1-forms on $M$. Note that since $Z_{0}, Z_{1}, Z_{2}$ span the tangent space to $M, \omega_{0}^{3}=0$ on $M$. Differentiating (1.1) gives the structure equations

$$
d \omega_{i}^{j}=\sum \omega_{i}^{k} \wedge \omega_{k}^{j}
$$

these are merely the structure equations of $G l(4)$, transferred to $M$. In particular, differentiating $\omega_{0}^{3}=0$ and applying the Cartan lemma (cf. [12]) gives us

$$
\omega_{\alpha}^{3}=\sum h_{\alpha \beta} \omega^{\beta}, \quad h_{\alpha \beta}=h_{\beta \alpha}, \quad 1 \leqslant \alpha, \beta \leqslant 2 .
$$

In classical Riemannian geometry, the quadratic form $\sum \omega_{\alpha}^{3} \otimes \omega^{\alpha}$ is well defined and is called the second fundamental form of $M$; in our case, since there is so much leeway in the "projective normal" $Z_{3}$, we have the following.

(1.3) Lemma. The (projective) second fundamental form

$$
\mathrm{II}=\Sigma \omega_{\alpha}^{3} \otimes \omega^{\alpha} \otimes Z_{3}=\sum h_{\alpha \beta} \omega^{\alpha} \otimes \omega^{\beta} \otimes Z_{3}
$$

is an intrinsically defined quadratic form on $M$ with values in the normal bundle $N_{M}$ of $M$ in $\mathbf{P}^{3}$.

Proof. Consider a change of frame field $Z_{i}^{*}=\sum a_{i}^{j} Z_{j}$ :

$$
\begin{aligned}
& Z_{0}^{*}=a_{0}^{0} Z_{0}, \\
& Z_{\alpha}^{*}=a_{\alpha}^{0} Z_{0}+\sum a_{\alpha}^{\beta} Z_{\beta}, \\
& Z_{3}^{*}=a_{3}^{0} Z_{0}+a_{3}^{1} Z_{1}+a_{3}^{2} Z_{2}+a_{3}^{3} Z_{3} .
\end{aligned}
$$

(Here the $a_{i}^{j}$ are functions on $M$.) Writing $\underline{a}=\left(a_{i}^{j}\right)$ and $\underline{\omega}=\left(\omega_{i}^{j}\right)$, the transformation rule for the connection matrix is given by [11]

$$
\omega^{*}=\underline{a} \underline{\omega} \underline{a}^{-1}+d \underline{a} \cdot \underline{a}^{-1} \text {. }
$$

Computing, we find

$$
\begin{aligned}
\sum \omega_{\alpha}^{3^{*}} \otimes \omega^{\alpha^{*}} \otimes Z_{3}^{*} & =\sum \omega_{i}^{3^{*}} \otimes \omega^{i^{*}} \otimes Z_{3}^{*} \quad \text { since } \omega_{0}^{3^{*}}=\omega^{3^{*}}=0 \\
& =\sum a_{i}^{j} \omega_{j}^{k}\left(a^{-1}\right)_{k}^{3} \otimes a_{0}^{l} \omega_{l}^{m}\left(a^{-1}\right)_{m}^{i} \otimes a_{3}^{q} Z_{q} \\
& =a_{0}^{0} \sum \omega_{j}^{k} \otimes \omega^{j} \otimes Z_{k} \\
& \equiv a_{0}^{0} \sum \omega_{\alpha}^{3} \otimes \omega^{\alpha} \otimes Z_{3}, \bmod \left(Z_{0}, Z_{1}, Z_{2}\right) .
\end{aligned}
$$

Since $Z_{3}$ is a section of $\mathbf{C}^{4} / \tilde{T} M$ and since $\left(a_{0}^{0}\right)^{-1}$ gives the transition functions for the tautological line bundle, we see that this is a well-defined quadratic form with values in

$$
\vartheta_{M}(1) \otimes \mathbf{C}^{4} / \tilde{T} M \cong N_{M},
$$


where we use the Euler sequence [23] to obtain the final isomorphism. ${ }^{1}$ q.e.d.

Define the projective Gauss mapping

$$
\begin{aligned}
\gamma: M & \rightarrow \mathbf{P}^{3^{*}} \\
z & \rightarrow \tilde{T}_{z} M .
\end{aligned}
$$

Lifting to the frame level, we compute its derivative. Since $\gamma(z)=Z_{0} \wedge Z_{1} \wedge$ $Z_{2}$, we have, using (1.1),

$$
\begin{aligned}
d \gamma \equiv \omega_{1}^{3}\left(Z_{0} \wedge Z_{3} \wedge Z_{2}\right)+\omega_{2}^{3}\left(Z_{0} \wedge Z_{1} \wedge Z_{3}\right), & \\
& \bmod \left(Z_{0} \wedge Z_{1} \wedge Z_{2}\right) .
\end{aligned}
$$

In particular, the rank of $\gamma$ is equal to the rank of the matrix $\left(h_{\alpha \beta}\right)$. As in classical surface theory, define $z \in M$ to be a parabolic point if $\operatorname{rank}\left(d \gamma_{z}\right)=$ $\operatorname{rank}\left(h_{\alpha \beta}\right) \leqslant 1$, a planar point if $d \gamma_{z}=0$. Define $v \in T_{z} M$ to be an asymptotic direction if $\operatorname{II}(v, v)=0$; we say $C \subset M$ is an asymptotic curve if its tangent vector at each point is an asymptotic direction. Note that at a nonplanar parabolic point $z$, there is a unique asymptotic direction, and it spans ker $d \gamma_{z}$.

It will be useful later on to have a concrete formula for the second fundamental form. Consider the case of a graph $M=\{z=f(x, y)\} \subset \mathbf{C}^{3} \subset$ $\mathbf{P}^{3}$. Working in affine coordinates, we take $Z_{1}=\left(1,0, f_{x}\right), Z_{2}=\left(0,1, f_{y}\right)$, $Z_{3}=(0,0,1), \omega^{1}=d x, \omega^{2}=d y$. From (1.1) it is easy to see that

$$
\mathrm{II}=f_{x x} d x^{2}+2 f_{x y} d x d y+f_{y y} d y^{2},
$$

i.e., the second fundamental form is given by the hessian of $f$. From this it is easy to deduce the following classical interpretation of the asymptotic directions.

(1.6) Lemma. At a nonplanar point $z \in M$, the tangent cone of $\tilde{\boldsymbol{T}}_{z} M \cap M$ consists precisely of the asymptotic lines in $\tilde{T}_{z} M$. In particular, at a parabolic point, this is a double line.

Proof. Take $z=(0,0,0)$ and $f_{x}(0)=f_{y}(0)=0$. Then the result is immediate. q.e.d.

Generally the parabolic points of $M$ form a curve $\Pi$, which is called the parabolic curve. Indeed, if we require that zero be a regular value of $K=$ $\operatorname{det}\left(h_{\alpha \beta}\right)$, then $\Pi$ will be smooth and contain no planar points. We next characterize those points of $\Pi$ at which the asymptotic direction is tangent to $\Pi$. Choose a frame $Z_{0} Z_{1} Z_{2} Z_{3}$ so that $Z_{1}$ is tangent to $\Pi=\left\{h_{11} h_{22}-h_{12}^{2}=0\right\}$. If $Z_{1}(P)$ is asymptotic, then we infer that $h_{11}(P)=h_{12}(P)=0$. From (1.4) it

\footnotetext{
${ }^{1}$ In [12], Griffiths and Harris actually have this wrong, but since all their work is local, the error is inconsequential. For us, however, it will prove essential in $\S 2$.
} 
now follows that $d \gamma_{P}\left(Z_{1}\right)=0$, whence $P$ is a critical point of $\gamma \mid \Pi$. Such a point $P$ is called a cusp of the Gauss map; the terminology arises from the geometry of the image curve $\gamma(\Pi) \subset \mathbf{P}^{3^{*}}$. Conversely, it is easy to see that if $P$ is a cusp, then the tangent vector to $\Pi$ at $P$ is asymptotic.

We shall call a cusp $P$ nondegenerate if the tangency of the asymptotic line field along $\Pi$ is nondegenerate at $P$. More specifically, choose an asymptotic vector field which is nonzero at $P$ and project it to $N_{\Pi}$; this section of $N_{\Pi}$ will then have a nondegenerate zero at $P$. We shall soon make this explicit in local coordinates.

We are now interested in characterizing the cusp locus more geometrically. For the rest of this section we consider surfaces $M \subset \mathbf{P}^{3}$ satisfying the properties:

(i) zero is a regular value of $K$ (in particular, $\Pi$ is smooth);

(ii) the cusps of the Gauss map are nondegenerate.

In $\$ 3$ we shall prove that the generic surface in $\mathbf{P}^{3}$ enjoys these properties. Consider the locus of inflection points of asymptotic curves in $M-\Pi$; let $\mathcal{T}$ be its closure in $M$. We call $\mathcal{T}$ the asymptotic flex curve; it may be reducible or singular. Our main result in this section is the following

(1.8) Theorem. Let $M \subset \mathbf{P}^{3}$ be a surface satisfying properties (1.7). Then the cusps of the Gauss map are precisely the points of intersection of $\Pi$ and $\sigma$. Moreover, $\mathcal{T}$ is smooth at these points, and $\Pi$ and $\mathcal{T}$ are simply tangent at these points.

In order to prove this theorem, it is convenient to analyze the geometry of an auxiliary surface $\tilde{M}$, the "asymptotic double cover" of $M$. It is to this task that we turn next. First of all, given an abstract surface, there is a natural isomorphism

$$
\mathbf{P}(T M) \cong \mathbf{P}\left(T^{*} M\right),
$$

with a line in $T M$ corresponding to its annihilator in $T^{*} M$. Now, given $M \subset \mathbf{P}^{3}$, define $M^{\prime} \subset \mathbf{P}(T M)$ by $M^{\prime}=\{v: \operatorname{II}(v, v)=0\}$, and let $\tilde{M} \subset \mathbf{P}\left(T^{*} M\right)$ be the corresponding surface under the isomorphism above.

More specifically, choose local coordinates (and corresponding frames) so that II $=\lambda d x^{2}+\mu d y^{2}$, and take $\Pi$ to be given by $\mu=0$. Clearly along $\Pi$ the asymptotic direction is $\partial / \partial y$ and so $P$ is a cusp of Gauss precisely when $\mu_{y}(P)=0$. Now, on $T^{*} M$ we have coordinates $x, y, \dot{x}, \dot{y}$ and, setting $p=\dot{y} / \dot{x}$ $(\dot{x} \neq 0)$, we obtain local coordinates $x, y, p$ on $\mathbf{P}\left(T^{*} M\right)$. In terms of these coordinates, define $\tilde{M} \subset \mathbf{P}\left(T^{*} M\right)$ by

$$
f(x, y, p)=\mu+\lambda p^{2}=0 .
$$


Note that $\tilde{M}$ is a smooth submanifold, for

$$
d f=d \mu+p^{2} d \lambda+2 p \lambda d p
$$

and when $p=0, d f=d \mu \neq 0$, since zero is a regular value of $K=\lambda \mu$. On the other hand, away from the parabolic curve in $M, \tilde{M} \stackrel{\pi}{\rightarrow} M$ is a double cover (corresponding to the geometric fact that the asymptotic directions are distinct). Indeed, lying over $P \notin \Pi$ are the two linearly independent 1 -forms $\eta_{1}, \eta_{2}$ (up to scalars) such that II $=\eta_{1} \otimes \eta_{2}$, i.e., the annihilators of the two asymptotic directions. As $P$ approaches a parabolic point, this degenerates to II $=\eta_{1} \otimes \eta_{1}$, as we expect. In sum, then, $\tilde{M} \stackrel{\pi}{\rightarrow} M$ is a branched double cover, branched over $\Pi$.

We now reinterpret the cusps of Gauss in terms of the geometry of $\tilde{M}$. Note that on $T^{*} M$ we have a canonically defined l-form $\Theta=\dot{x} d x+\dot{y} d y$ which induces a contact structure on $\mathbf{P}\left(T^{*} M\right)$, given locally by $\theta=d x+p d y$.

(1.10) Lemma. $A$ cusp point $P$ is a zero of $\left.\theta\right|_{\tilde{M}}$. Moreover, condition (ii) of (1.7) implies that $P$ is a nondegenerate zero of $\theta$, i.e., that $\theta$ is transverse to the zero section of $T^{*} \tilde{M}$ at $P$.

Proof. (Note that we are identifying points of $\pi^{-1}(\Pi)=\tilde{\Pi}$ with their images in $\Pi$.) When $p=0, d f=d \mu \equiv 0 \bmod (d x)$ at a cusp $P$, so $T_{P} \tilde{M}=$ $\operatorname{ker} d f_{P}=\operatorname{ker} \theta_{P}$. To prove the last equality, we rewrite $\theta$ in terms of local coordinates on $\tilde{M}$. Since $\mu_{x}(P) \neq 0$, it follows from the implicit function theorem that in a neighborhood of $P$ we may write $x=x(y, p)$ on $\tilde{M}$. Then

$$
\boldsymbol{\theta}=A(y, p) d y+B(y, p) d p,
$$

where

$$
A(y, p)=p-\frac{\mu_{y}+p^{2} \lambda_{y}}{\mu_{x}+p^{2} \lambda_{x}}, \quad B(y, p)=-\frac{2 p \lambda}{\mu_{x}+p^{2} \lambda_{x}} .
$$

Letting $P \leftrightarrow(y, p)=(0,0)$, we see that $\theta$ is transverse to the zero section of $T^{*} \tilde{M}$ at $P$ if and only if the jacobian determinant $\partial(A, B) /\left.\partial(y, p)\right|_{(0,0)} \neq 0$. Since $P$ is a cusp, $\mu_{y}(0)=0$; since $P$ is nondegenerate, $\mu_{y y}(0) \neq 0$. Using these data, we find that

$$
\left.\frac{\partial(A, B)}{\partial(y, p)}\right|_{(0,0)}=\frac{2 \lambda(0) \mu_{y y}(0)}{\mu_{x}^{2}(0)} \neq 0,
$$

as required. Note that, conversely, if a parabolic point $P$ is a zero of $\theta$, then $P$ is a cusp. q.e.d.

Having given a geometric characterization of cusps in $\tilde{M}$, we proceed to discuss the asymptotic flex curve as it appears in $\tilde{M}$. 
(1.11) Lemma. Let $\tilde{\mathfrak{T}}=\{A(y, p)=0\} \subset \tilde{M}$. Then $\pi(\tilde{\mathfrak{T}})=\sigma$.

Proof. The main point here is that integral curves of $\theta=0$ in $\tilde{M}$ project to asymptotic curves in $M$. Moreover, the $p$-coordinate gives the negative reciprocal of the slope downstairs (this is due to the presence of duality in the isomorphism (1.9)). Since an inflection point of a plane curve is a critical point of its slope function, we see that asymptotic flexes correspond to points on the integral curves of $\theta=0$ where $d p / d y=0$. Since $\theta=A d y+B d p$, this means we require $d p / d y=-A / B$, and the lemma follows.

Proof of Theorem (1.8). From Lemmas (1.10) and (1.11) we infer that $\tilde{\sigma} \cap\{p=0\}$ consists precisely of points such that $\mu_{y}=0$, i.e., cusps of Gauss. We can, however, easily compute more. In $(y, p)$ coordinates at a cusp (which we take to be the origin), the slope of $\tilde{\mathcal{T}}$ is

$$
\frac{d p}{d y}=-\left.\frac{\partial A / \partial y}{\partial A / \partial p}\right|_{(0,0)}=\frac{\mu_{y y}(0)}{\mu_{x}(0)},
$$

which is neither 0 nor $\infty$. Since $\pi: \tilde{M} \rightarrow M$ ramifies precisely along $\tilde{\Pi}$, with ker $d \pi$ spanned by $\partial / \partial p$, we deduce that $\sigma=\pi(\tilde{\sigma})$ is simply tangent to $\Pi$. q.e.d.

In the proof of Lemma (1.11) we sought points with "horizontal tangents" in $(p, y)$-coordinates. In terms of the projective connection on $M$ with which we have been working, this can be phrased completely intrinsically. Consider the bundle $F(M)$ of adapted frames on $M$ with the intermediate projection:

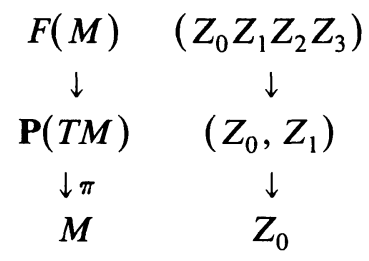

It is easy to check that the forms $\omega^{1}, \omega^{2}, \omega_{1}^{2}$ on $F(M)$ are horizontal for the projection $F(M) \rightarrow \mathbf{P}(T M)$; we then define the subspace $\omega_{1}^{2}=0$ to be horizontal for the projection $\mathbf{P}(T M) \stackrel{\pi}{\rightarrow} M$ (and similarly for the restriction of $\pi$ to $\tilde{M}$ ). That this gives the right notion is clear: let $\{Z(t)\} \subset M$ be an immersed curve, and let $\tilde{Z}(t)=\left(Z_{0}=Z(t), Z_{1}=Z^{\prime}(t)\right)$ be the canonical lift to $\mathbf{P}(T M)$; then $Z(0)$ is an inflection point precisely when $Z^{\prime \prime}(0) \equiv 0 \bmod \left(Z(0), Z^{\prime}(0)\right)$, i.e., when $\omega_{1}^{2}\left(Z^{\prime}(0)\right)=0$.

Transferring this analysis to $\mathbf{P}\left(T^{*} M\right)$, using the isomorphism (1.9) enables us to give an intrinsic description of the curve $\tilde{\mathcal{T}} \subset \tilde{M}$. Let $\tilde{M} \subset \mathbf{P}\left(T^{*} M\right)$ be defined by the equation $f=0$. (Recall that viewed in $\mathbf{P}(T M), \tilde{M}$ is cut out by 
the global equation $\operatorname{II}(v, v)=0$.) For future reference, we point out that in local coordinates $x, y, p$, if

$$
\mathrm{II}=\lambda d x^{2}+2 \sigma d x d y+\mu d y^{2}
$$

then

$$
f(x, y, p)=\lambda p^{2}-2 \sigma p+\mu .
$$

Then we claim that $\tilde{\mathscr{T}}$ is cut out on $\tilde{M}$ by the equation

$$
d f \wedge \theta \wedge \omega_{1}^{2}=0
$$

on $\mathbf{P}\left(T^{*} M\right)$. For recall that $x \in \tilde{\mathfrak{J}}$ precisely when there is a nonzero tangent vector $v$ at $x \in \tilde{M}$ which satisfies $d f(v)=\theta(v)=\omega_{1}^{2}(v)=0$. Moreover, in local coordinates, $\omega_{1}^{2}$ is a multiple of $d p$, and this equation reduces to

$$
p f_{x}-f_{y}=0,
$$

which is the identical equation to that which we derived earlier for $\tilde{\widetilde{T}}$ in Lemma (1.11).

Remark. Using the geometry of the asymptotic double cover, we may define a modulus $\alpha$ of a generic Gaussian cusp as follows. Suppose $P$ is a cusp of the Gauss map of $M \subset \mathbf{P}^{3}$, and $M$ satisfies (1.7) near $P$. Let $\tilde{P}$ be the corresponding point of $\tilde{M}$. Consider the following five lines in $T_{\tilde{P}} \tilde{M}$. Let $L_{1}$ be the tangent line of the curve $\tilde{\Pi}$ at $\tilde{P}$. Let $L_{2}$ be the tangent line of the curve $\tilde{\mathcal{T}}$ at $\tilde{P}$. Let $L_{3}$ be the kernel of $d \pi_{\tilde{P}}$. Let $L_{4}$ and $L_{5}$ be the tangents to the two branches of the integral curve of $\theta=0$ through $\tilde{P}$. Set $\alpha$ equal to the sum of the cross-ratios of the sets $\left(L_{1}, L_{2}, L_{3}, L_{4}\right)$ and $\left(L_{1}, L_{2}, L_{3}, L_{5}\right)$. Then $\alpha$ is invariant under projective transformations of $\mathbf{P}^{3}$. The existence of such an invariant was suggested to us by $\mathbf{R}$. Varley.

For example, consider the germs at 0 of the (real or complex) surfaces

$$
z=x^{2}+x y^{2}+c y^{4} \quad(c \in \mathbf{R} \text { or } \mathbf{C})
$$

considered (over R) by Platonova [21] and Banchoff et al. [6]. A theorem of Platonova [21, Theorem 1] (see also [4, p. 126]) implies that if a generic $C^{\infty}$ surface $M$ in $\mathbf{R} P^{3}$ has a Gaussian cusp at $P$, then the 4-jet of $M$ at $P$ is projectively equivalent to one of the above germs. These germs satisfy (1.7) if and only if $c \neq 1 / 4$, and then $\alpha=5 / 8 c$, so all these germs are projectively distinct.

\section{Global theory}

Up to this point all our arguments have been local. We now restrict ourselves to the case of a complex algebraic surface in $\mathbf{P}^{3}$ in order to use (global) algebro-geometric arguments to count the number of cusps of the 
Gauss map. The computation is based on the algebra of the projective second fundamental form.

(2.1) Lemma. $\Pi$ belongs to the linear system $|(4 d-8) H|$ on $M$.

Proof. Recall that $\Pi=\{K=0\}$, where $K=\operatorname{det}$ II. Viewing II as a section of $\operatorname{Sym}^{2}\left(T^{*} M\right) \otimes N$, det II gives a section of $\left(K_{M} \otimes N\right)^{2}$. Now standard adjunction formulas from algebraic geometry [11] give $K_{M} \in|(d-4) H|$ and $N \in|d H|$, so $(K \otimes N)^{2} \in|2(2 d-4) H|$. q.e.d.

Let $\varrho \rightarrow \tilde{M}$ be the restriction of the tautological line bundle on $\mathbf{P}\left(T^{*} M\right)$; let $\mathcal{L}^{\prime} \subset \pi^{*} T M$ be the subbundle on $\tilde{M}$ whose fibre over $\tilde{x}$ is the asymptotic line in $T_{x} M$ which corresponds to $\tilde{x}$. More formally, $\mathscr{L}^{\prime}=\operatorname{Ann}(\mathcal{L})$, and standard linear algebra gives

$$
\pi^{*} T M / \varrho^{\prime} \cong \varrho^{*} .
$$

(2.3) Lemma. On $\Pi \cong \tilde{\Pi}$ we have the fundamental exact sequence

$$
0 \rightarrow \mathcal{L}^{\prime} \rightarrow T M \stackrel{\text { II }}{\rightarrow} N \otimes \mathcal{L} \rightarrow 0 \text {. }
$$

Proof. Let $P$ be a parabolic point, and let $w_{0} \in T_{P} M$ be an asymptotic vector. Then $\mathrm{II}\left(v, w_{0}\right)=0$ for all $v \in T_{P} M$. Now II induces a surjective mapping

$$
T M \rightarrow N \otimes \mathcal{L}, \quad v \rightarrow \mathrm{II}(v, \cdot),
$$

as an element of $N \otimes \mathcal{L}$ is a 1 -form with values in $N$ which annihilates the subbundle $\mathcal{L}^{\prime} \subset T M$. Moreover, the kernel of this mapping is clearly $\mathfrak{L}^{\prime}$, finishing the proof.

(2.4) Lemma. If $M$ has properties (1.7)(i), (ii), then \#(cusps) = $c_{1}(N(\Pi, M))-c_{1}\left(\left.\mathcal{L}^{\prime}\right|_{\Pi}\right)$, where $N(\Pi, M)$ is the normal bundle of $\Pi$ in $M$.

Proof. Let $N=N(\Pi, M)=\left.T M\right|_{\Pi} / T \Pi$, and let $p:\left.T M\right|_{\Pi} \rightarrow N$ be the projection homomorphism. The homomorphism $\left.p\right|_{\mathfrak{e}^{\prime}}$ defines a section of the line bundle $\operatorname{HOM}\left(\mathcal{L}^{\prime}, N\right)$, and the zeros of this section are just the cusps of the Gauss map. Therefore

$$
\#(\text { cusps })=c_{1}\left(\operatorname{HOM}\left(\mathcal{L}^{\prime}, N\right)\right)=c_{1}\left(\left(\varrho^{\prime}\right)^{*} \otimes N\right)=c_{1}(N)-c_{1}\left(\varrho^{\prime}\right) \text {. q.e.d }
$$

We now assemble these facts to prove the

(2.5) Theorem. Let $M \subset \mathbf{P}^{3}$ be a complex algebraic surface of degree $d$ satisfying (1.7)(i)(ii). Then there are precisely $2 d(d-2)(11 d-24)$ cusps of the Gauss map.

Proof. By (2.4), \#(cusps) $=c_{1}(N(\Pi, M))-c_{1}\left(\mathcal{L}^{\prime}\right)=\Pi \cdot \Pi-c_{1}\left(\mathcal{L}^{\prime}\right)$. Taking Chern classes of the exact sequence in (2.3) and using (2.2), we obtain the relation

$$
c_{1}\left(\mathfrak{L}^{\prime}\right)=c_{1}\left(\left.T M\right|_{\Pi}\right)-\frac{1}{2} c_{1}\left(\left.N\right|_{\Pi}\right)=-K_{M} \cdot \Pi-\frac{1}{2} c_{1}\left(\left.N\right|_{\Pi}\right) .
$$


Therefore, using (2.1), we have

$$
\begin{aligned}
\#(\text { cusps }) & =\Pi \cdot \Pi+K_{M} \cdot \Pi+\frac{1}{2} c_{1}\left(\left.N\right|_{\Pi}\right) \\
& =d(4 d-8)\left\{(4 d-8)+(d-4)+\frac{1}{2} d\right\} \\
& =2 d(d-2)(11 d-24) . \text { q.e.d. }
\end{aligned}
$$

We apply the preceding theory to the simplest examples at hand. Before beginning, however, it will be convenient to give a classical geometric interpretation of the asymptotic flex locus. A line $L$ and a surface $M$ in $\mathbf{P}^{3}$ are said to have $k$-point contact at $P \in M$ if the intersection multiplicity at $P$ of $L$ and $M$ is equal to $k$. (If $L$ is contained in $M$, then $L$ and $M$ are said to have infinite contact at each $P \in L$.) Working in affine coordinates $x, y, z$ with $L=\{y=z$ $=0\}, M=\{g=0\}$ and $P=(0,0,0)$, the algebraic definition of intersection multiplicity is

$$
\operatorname{dim}_{\mathbf{C}} \mathbf{C}[x, y, z]_{0} /(g, y, z),
$$

i.e., the length of the ideal $(g, y, z)$ in the local ring $\mathbf{C}[x, y, z]_{0}$, which is equal to

$$
\operatorname{dim}_{\mathbf{C}} \mathbf{C}[x]_{0} /(g(x, 0,0)),
$$

and this is equal to the number $k$ such that

$$
\frac{\partial^{k} g}{\partial x^{k}}(0) \neq 0 \quad \text { and } \quad \frac{\partial^{i} g}{\partial x^{i}}(0)=0, \quad i=0, \ldots, k-1 .
$$

We now relate this concept to asymptotic flexes. We shall say that $L$ is an asymptotic flex line of $M$ at $P$ if $L$ is an asymptotic tangent line of $M$ at $P$, and the asymptotic curve through $P$ in the direction $L$ has a flex at $P$.

(2.6) Proposition. Suppose that the line $L$ and the surface $M$ have $k$-point contact at $P \in M$. Then $L$ is an asymptotic tangent line to $M$ at $P$ if and only if $k \geqslant 3$. If $P$ is not a parabolic point, then $L$ is an asymptotic flex line of $M$ at $P$ if and only if $k \geqslant 4$.

Proof. We may assume $P$ is the origin in $\mathbf{C}^{3}$ and that $M=\{z=f(x, y)\}$ with $f_{x}(0)=f_{y}(0)=0$. Let $L$ be the $x$-axis. By (1.5), the second fundamental form of $M$ is given by the hessian of $f$, if we use the framing $Z_{1}=\left(1,0, f_{x}\right)$, $Z_{2}=\left(0,1, f_{y}\right), Z_{3}=(0,0,1)$. Let $\alpha(t)$ be a curve on $M$ with $\alpha(0)=0$ and $\alpha^{\prime}(0)=(1,0,0)$. If $\alpha^{\prime}(t)=\beta_{1} Z_{1}+\beta_{2} Z_{2}$, then $\beta_{1}(0)=1$ and $\beta_{2}(0)=0$. Then

$$
\begin{aligned}
\alpha^{\prime \prime}(t) & =\beta_{1} Z_{1}^{\prime}+\beta_{1}^{\prime} Z_{1}+\beta_{2} Z_{2}^{\prime}+\beta_{2}^{\prime} Z_{2} \\
& =\beta_{1}^{\prime} Z_{1}+\beta_{2}^{\prime} Z_{2}+\left(\beta_{1}^{2} f_{x x}+2 \beta_{1} \beta_{2} f_{x y}+\beta_{2} f_{y y}\right) Z_{3} ;
\end{aligned}
$$

setting $t=0$ we find $\alpha^{\prime \prime}(0)=\beta_{1}^{\prime}(0) Z_{1}+\beta_{2}^{\prime}(0) Z_{2}+f_{x x}(0) Z_{3}$. Therefore $f_{x x}(0)$ $=0$ if and only if the curve $\alpha(t)$ is asymptotic at 0 . Moreover, assuming the 
curve $\alpha(t)$ is asymptotic, the coefficient of $Z_{3}$ is identically zero for all $t$. Differentiating this coefficient, we find that $2 \beta_{1}^{\prime}(0) f_{x x}(0)+f_{x x x}(0)+$ $2 \beta_{2}^{\prime}(0) f_{x y}(0)=0$. Now $f_{x x}(0)=0$, so if we assume that the origin is not a parabolic point, then $f_{x y}(0) \neq 0$. Thus $f_{x x x}(0)=0$ if and only if $\beta_{2}^{\prime}(0)=0$, if and only if $\alpha^{\prime \prime}(0)$ is parallel to $\alpha^{\prime}(0)$, i.e., 0 is an inflection point of $\alpha(t)$, as required.

The generic cubic. Let us now consider a generic cubic surface $M \subset \mathbf{P}^{3}$. According to Theorem (2.5), we expect 54 cusps of the Gauss map. Of course these must derive somehow from the 27 lines on $M$. First of all, we claim that the curve $\mathcal{T}$ of asymptotic flexes consists precisely of the 27 lines. By Proposition (2.6), the tangent line to an asymptotic curve at a flex must have four-point contact with $M$. Since $M$ is a cubic, this line must lie completely in $M$. On the other hand, any line contained in a surface clearly is an asymptotic curve and consists entirely of flex points. Now the result follows from Theorem (1.8) and the following proposition.

(2.7) Proposition. Each line on a cubic surface meets the parabolic curve exactly twice, and it is tangent to the parabolic curve at both of the intersection points.

Proof. Let $L$ be a line on the cubic surface $M$, and let $H$ be a plane containing $L$. Then $H \cap M$ is a cubic curve containing $L$, so $H \cap M=L \cup C$, where $C$ is a conic. ( $C$ may be reducible, but $L \cup C$ cannot contain a double line.) Now the intersection number of $L$ and $C$ is 2 , and $H$ is tangent to $M$ at each point of $L \cap C$. If these two points coincide, i.e., if $L$ is tangent to $C$, then this point is a parabolic point of $M$, by Lemma (1.6).

Now define an involution $\sigma$ of $L$ as follows. Given $p \in L, T_{p} M \cap M$ is tangent to $M$ at another point $q$, and we set $\sigma(p)=q$. The involution $\sigma$ is algebraic, and by the Lefschetz fixed point theorem, $\sigma$ has two fixed points, which are the two parabolic points on $L$.

The Fermat cubic. Having discussed the case of the generic cubic surface, we would like next to specialize to the Fermat cubic $x_{0}^{3}+x_{1}^{3}+x_{2}^{3}+x_{3}^{3}=0$. The parabolic curve is given by the hessian, i.e., $\Pi$ is cut out on $M$ by $x_{0} x_{1} x_{2} x_{3}=0$, so $\Pi$ is a reducible curve with $\left(\begin{array}{l}4 \\ 2\end{array}\right) \cdot 3=18$ nodes. (Thus property (1.7)(i) fails.) It is a straightforward calculation that the restriction of the Gauss map $\gamma$ to a smooth component of $\Pi$ has critical points only at the 9 nodes on that component. As in the generic case, the asymptotic flex curve consists of the 27 lines. Each line passes through two nodes, and through each node pass three lines, so the intersection number $\Pi \cdot \mathcal{T}=108$. This is what we expect from the generic case, where $\Pi$ and $\mathcal{T}$ meet 54 times, and each intersection point has multiplicity 2 . If we perturb the Fermat cubic, each of 
the 18 nodes of $\Pi$ will produce 3 cusps of the Gauss map. (A local description of the real locus of such a perturbation is given by [6, Example 3a, p. 17]; cf. also [15, §14, Figure 8].)

It is interesting to examine the Fermat cubic from the viewpoint of the geometry of $\tilde{M}$ and $\tilde{\mathscr{T}}$ encountered in the proof of Theorem (1.8). Working in the set $x_{0}=1$, and in a neighborhood of $(x, y, z)=(0,0,-1)$ (one of the nodes of $\Pi$ ) we can write $z=-\left(1+x^{3}+y^{3}\right)^{1 / 3}$. Then we may take the second fundamental form to be

$$
\mathrm{II}=x\left(1+y^{3}\right) d x^{2}-2 x^{2} y^{2} d x d y+y\left(1+x^{3}\right) d y^{2} .
$$

It follows from (1.12) that the equation of $\tilde{M}$ in $\mathbf{P}\left(T^{*} M\right)$ is

$$
f(x, y, p)=y\left(1+x^{3}\right)+2 x^{2} y^{2} p+x\left(1+y^{3}\right) p^{2}=0 \text {. }
$$

We see that $\tilde{M}$ is smooth, and that in fact at the nodes of $\Pi, \tilde{M}$ is the blow-up of $M$. By (1.14), $\tilde{\sigma}$ is cut out on $\tilde{M}$ by the equation $p f_{x}-f_{y}=0$. The line $y=\omega x, p=-1 / \omega$, satisfies this equation if and only if $\omega^{3}=-1$. (Because $p$ is a coordinate on $\mathbf{P}\left(T_{0}^{*} M\right)$, it is the negative reciprocal of the slope in $T_{0} M$.) Thus the lines $(t, \omega t,-1), \omega^{3}=-1$, lie in $\mathcal{T}$, as we expected. To see that these three lines are the only components of $\mathcal{T}$ at $(x, y, z)=(0,0,-1)$, we proceed as follows. Let $\Phi=\left\{p f_{x}-f_{y}=0\right\}$, the surface in $\mathbf{P}\left(T^{*} M\right)$ such that $\Phi \cap \tilde{M}=\tilde{\Im}$. Let $\tilde{M}^{\prime}, \Phi^{\prime}, \tilde{\mathfrak{丁}}^{\prime} \subset T^{*} M$ be the inverse images of $\tilde{M}, \Phi, \tilde{\Im}$, respectively. In coordinates $(x, y, \dot{x}, \dot{y})$ on $T^{*} M$, the tangent cone to $\Phi^{\prime}$ at 0 is $\dot{x}^{3}-\dot{y}^{3}=0$ (or $p^{3}=1$ ), so the tangent cone of $\tilde{\mathscr{T}}$ is a subscheme of this locus. (Here we are using the fact that the tangent cone of $X \cap Y$ at $x$ is a subscheme of the intersection of the tangent cone of $X$ at $x$ and the tangent cone of $Y$ at $x$.) It follows that the components of $\mathscr{T}$ at $(x, y, z)=(0,0,-1)$ are precisely the three lines found above.

The Fermat quartic. Let us consider last the example of the Fermat quartic $x_{0}^{4}+x_{1}^{4}+x_{2}^{4}+x_{3}^{4}=0$. While it certainly fails to meet the requirements (1.7) we imposed on our surfaces (indeed, we shall soon see to what extent!), we are still able to compute that $\Pi \cdot \mathcal{T}=640$, twice the number of cusps of the Gauss map given by Theorem (2.5). Note, first of all, that the parabolic curve $\Pi$ is unreduced, cut out on the quartic $M$ by the surface $x_{0}^{2} x_{1}^{2} x_{2}^{2} x_{3}^{2}=0$. The underlying reduced curve has $\left(\begin{array}{l}4 \\ 2\end{array}\right) \cdot 4=24$ nodes. Through each node of $\Pi$ pass four lines on $M$; each line passes through two nodes, and so there are $\frac{1}{2} \cdot 24 \cdot 4=48$ lines. They certainly comprise part of the asymptotic flex curve $\mathcal{T}$. It is reasonable to expect that the parabolic curve will also be part of $\mathcal{T}$ : since $\Pi$ is unreduced, the restriction of the Gauss map to $\Pi$ is singular at every 
point of $\Pi$. We claim that $\mathcal{T}$ consists of the 48 lines together with the parabolic curve:

$$
\mathcal{T}=\Pi+\sum_{j=1}^{48} L_{j},
$$

so

$$
\Pi \cdot \mathcal{T}=\Pi \cdot\left(\Pi+\sum L_{i}\right)=\Pi \cdot \Pi+\Pi \cdot\left(\sum L_{i}\right)=4 \cdot 64+8 \cdot 48=640,
$$

as expected.

To justify the claim (2.9), we revert to the local differential geometric calculations. Working with $x_{0}=1$, near the node $(x, y, z)=(0,0, \sqrt{i})$, we have the second fundamental form

$$
\mathrm{II}=x^{2}\left(1+y^{4}\right) d x^{2}-2 x^{3} y^{3} d x d y+y^{2}\left(1+x^{4}\right) d y^{2}
$$

Neglecting terms of order greater than 2 (i.e., looking at the tangent cone of $\tilde{M}^{\prime}$ in $T^{*} M$ ), we see that in this case $\tilde{M}$ is singular, consisting of two branches which intersect along the $p$-axis and along the $x$-axis. In $P\left(T^{*} M\right)$, the equation of the tangent cone of $\tilde{M}^{\prime}$ becomes $x^{2} p^{2}+y^{2}=0$. A calculation shows that the equation of the tangent cone of $\Phi^{\prime}$ is $x p^{3}+y=0$. So the tangent cone of $\tilde{\mathfrak{g}}^{\prime}=\tilde{M}^{\prime} \cap \Phi^{\prime}$ is a subscheme of the locus $p^{2} x^{2}\left(1+p^{4}\right)=0$. This yields the four lines mentioned above $\left(y=\omega x, p=1 / \omega, \omega^{4}=-1\right)$, plus the $x$ and $p$ axes, each taken with multiplicity two. (Each of these loci is easily seen to be a subscheme of $\tilde{\widetilde{J}}$.) The $p$ axis projects to zero in $M$, but the $x$ axis projects to $\Pi$, as claimed. (Note that the unreduced $\Pi$ appears in $\mathcal{T}$. We see that this occurs because $\Phi$ intersects each of the two branches of $\tilde{M}$.)

Our discussion of the Fermat cubic and Fermat quartic indicates that the intersection number of the parabolic curve $\Pi$ and the asymptotic flex curve $\mathcal{T}$ should always be equal to $4 d(d-2)(11 d-24)$, for a smooth surface $M$ in $\mathbf{P}^{3}$ of degree $d$. An obvious requirement, however, is that $\Pi$ and $\mathcal{T}$ be 1-dimensional; i.e., not every point on $M$ is parabolic, and not every point of $M$ is an asymptotic flex. This merely rules out the plane and the quadric:

(2.10) Lemma. Let $M \subset \mathbf{P}^{3}$ be smooth. Then $M$ consists entirely of parabolic points only if $M$ is a plane; $M$ consists entirely of asymptotic flex points only if $M$ is a quadric surface.

Proof. (a) Suppose that the rank of the Gauss map $\gamma$ is equal to one on an open set of $M$. In this set, choose a frame for $M$ with $Z_{1} \in \operatorname{ker} d \gamma$. From (1.4) we have $\omega_{1}^{3} \equiv 0$ and $\omega_{2}^{3} \equiv 0 \bmod \left(\omega^{2}\right)$. From the symmetry of the second fundamental form, it follows that $\omega_{1}^{3}=0$. Using the structure equations (1.2), we find that $\omega_{1}^{2} \equiv 0 \bmod \left(\omega^{2}\right)$, and so integral curves of $Z_{1}$ are lines in $M . M$ is a developable ruled surface, and is therefore a plane, cone, or tangent surface to a space curve (cf. [12], [24]); only a plane is smooth. 
(b) Suppose $M$ consists entirely of asymptotic flex points. Then the asymptotic curves must be lines and so $M$ is ruled. Now we apply the adjunction formula to a line $C \subset M$. First of all, $K_{M}=(d-4) H$, where $d=$ degree $M$, and so $K_{C}=\left.(d-4) H \otimes[C]\right|_{C}$. Computing degrees of bundles on $C \cong \mathbf{P}^{1}$, we have $-2=d-4+C \cdot C$, so $C \cdot C=2-d$. For $C$ to be a ruling, it is necessary that $C \cdot C=0$, whence $d=2$. q.e.d.

The example of the Fermat quartic shows that in order to get the expected answer for the intersection number $\Pi \cdot \mathcal{T}$, both curves must be defined as schemes: their multiplicities are important. But such definitions arose naturally in $\S 1$. If $\tilde{M} \subset \mathbf{P}\left(T^{*} M\right)$ is defined by the equation $f=0$, then $\tilde{\Pi}$ is the intersection of $\tilde{M}$ with the surface

$$
\Psi=\left\{d f \wedge \omega^{1} \wedge \omega^{2}=0\right\},
$$

and $\Pi$ is the projection of $\tilde{\Pi}$ to $\tilde{M}$. The curve $\tilde{\widetilde{T}}$ is the intersection of $\tilde{M}$ with the surface

$$
\Phi=\left\{d f \wedge \theta \wedge \omega_{1}^{2}=0\right\}
$$

in $\mathbf{P}\left(T^{*} M\right)$ (1.13), and $\mathcal{T}$ is the projection of $\tilde{\mathcal{T}}$ to $M$. So $\Pi$ and $\mathcal{T}$ are defined as proper subschemes of $M$, provided $M$ has degree at least 3.

(2.11) Theorem. Let $M \subset \mathbf{P}^{3}$ be a smooth algebraic surface of degree $d \geqslant 3$. The intersection number of the parabolic curve $\Pi$ and the asymptotic flex curve $\mathcal{T}$ is equal to $4 d(d-2)(11 d-24)$.

Proof. Let $n=4 d(d-2)(11 d-24)$. By Theorems (1.8) and (2.5), $\Pi \cdot \sigma$ $=n$ if $M$ satisfies (1.7): (i) zero is a regular value of $K$ and (ii) the cusps of the Gauss map are nondegenerate. In Theorem (3.1) we shall prove that, for $d \geqslant 2$, the set of surfaces satisfying (1.7) is a nonempty Zariski open subset of the moduli space of smooth surfaces in $\mathbf{P}^{3}$ of degree $d$. Suppose $M_{0} \subset \mathbf{P}^{3}$ is a surface of degree $d$ which does not satisfy (1.7). Then for a generic line $L$ through $M_{0}$ in the moduli space of all surfaces of degree $d$ (which is a projective space), a punctured neighborhood of $M_{0}$ in $L$ will consist of surfaces which satisfy (1.7). So we have the following situation: $\left\{M_{t}\right\}_{t \in \Delta}$ is an analytic family of degree $d$ surfaces, parametrized by the unit disc $\Delta$, and for $t \in \Delta^{*}=$ $\Delta-\{0\}, M_{t}$ satisfies (1.7), so $\Pi_{t} \cdot \sigma_{t}=n$ in $M_{t}$. We wish to show that $\Pi_{0} \cdot \mathscr{T}_{0}=n$ in $M_{0}$.

To this end, consider the family of 3 -folds $X \stackrel{h}{\rightarrow} \Delta, h^{-1}(t)=\mathbf{P}\left(T^{*} M_{t}\right)$, together with the three families of hypersurfaces $Y_{i} \stackrel{h_{i}}{\rightarrow} \Delta, i=1,2,3: h_{1}^{-1}(t)=\tilde{M}_{t}$, $h_{2}^{-1}(t)=\Psi_{t}, h_{3}^{-1}(t)=\Phi_{t}$. The fibering theorem of J. King for holomorphic maps [14, Theorem 3.3.2] implies that for each $i$, the currents defined by $h_{i}^{-1}(0)$ 
and $h_{i}^{-1}(t), t \in \Delta^{*}$, are homologous in $Y_{i}$, and therefore in $X$. Now $\Pi_{t}=$ $\left(\pi_{t}\right)_{*}\left(h_{1}^{-1}(t) \cdot h_{2}^{-1}(t)\right)$ and $\mathcal{T}_{t}=\left(\pi_{t}\right)_{*}\left(h_{1}^{-1}(t) \cdot h_{3}^{-1}(t)\right)$ (intersection in $\mathbf{P}\left(T^{*} M_{t}\right)$ ). Therefore the currents defined by $\Pi_{0}$ and $\Pi_{t}, t \in \Delta^{*}$, are homologous, as are the currents defined by $\mathcal{T}_{0}$ and $\mathcal{T}_{t}, t \in \Delta^{*}$. (Note that the family $\left\{\mathbf{P}\left(T^{*} M_{t}\right)\right\}$ is topologically trivial). So $\Pi_{0} \cdot \mathcal{T}_{0}=\Pi_{t} \cdot \mathcal{T}_{t}, t \in \Delta^{*}$.

\section{Genericity}

In this section we will show that properties (1.7)(i) and (ii) are generic properties of complex algebraic surfaces in $\mathbf{P}^{3}$. More precisely, let $\Re_{d}$ be the moduli space of smooth surfaces of degree $d$ in $\mathbf{P}^{3}$.

(3.1) Theorem. For each integer $d \geqslant 2$, the set of degree $d$ surfaces with properties (1.7)(i), (ii) is a nonempty Zariski open subset of $\Re_{d}$.

The key of proving the openness part of this result is a proposition of Mather on local stability of morphisms of varieties [19]. First we recall the classical construction of the dual variety of $M$.

Let $M$ be a smooth surface in $\mathbf{P}^{3}$. Let $\left(\mathbf{P}^{3}\right)^{*}$ be the dual projective space, and let $\Gamma \subset M \times\left(\mathbf{P}^{3}\right)^{*}$ be the correspondence

$$
\Gamma=\{(x, H): x \in H\} .
$$

The variety $\Gamma$ is a smooth 4 -fold, since it is a bundle over $M$ with fiber $\{H \mid x \in H\} \cong \mathbf{P}^{2}$. Let

$$
f=f_{M}: \Gamma \rightarrow\left(\mathbf{P}^{3}\right)^{*}
$$

be projection to the second factor. The singular locus $\Sigma(f)$ is $\{(x, H): H$ is tangent to $M$ at $x$, which we identify with $M$ by projection to the first factor. The restriction of $f$ to $\Sigma(f) \cong M$ is the Gauss map $\gamma(x)=f\left(x, \tilde{T}_{x} M\right)=\tilde{T}_{x} M$, and the image of the Gauss map is the dual variety of $M$.

(3.3) Lemma. The set of degree $d$ surfaces $M$ such that $f_{M}$ is locally stable at each point of $\Gamma$ is a Zariski open subset of $\Re_{d}$.

An elementary discussion of local (infinitesimal) stability for $C^{\infty}$ maps can be found in [10, p. 73]. The complex algebraic version of local stability is presented in [19, p. 179].

Proof of (3.3). Let $\mathcal{E} \subset \mathbf{P}^{3} \times\left(\mathbf{P}^{3}\right)^{*} \times \mathfrak{N}_{d}$ be the subvariety $\varepsilon=$ $\{(x, H, M) \mid x \in H \cap M\}$, and let

$$
F: \mathcal{E} \rightarrow\left(\mathbf{P}^{3}\right)^{*} \times \Re_{d},
$$


$F(x, H, M)=(H, M)$. The map $F$ is just the family of maps $f_{M}$, parametrized by $\mathfrak{K}_{d}$. By [19, Proposition 1, p. 180], the set of $M \in \mathfrak{N}_{d}$ such that $f_{M}$ is locally stable at each finite subset of $\Gamma$ is Zariski open, provided that the maps $\Sigma(F) \rightarrow\left(\mathbf{P}^{3}\right)^{*} \times \Re_{d}$ and $\left(\mathbf{P}^{3}\right)^{*} \times \Re_{d} \rightarrow \Re_{d}$ are projective. A projective map $f: X \rightarrow Y$ is one which factors as the composition of an embedding of $X$ onto a closed subvariety of $\mathbf{P}^{N} \times Y$, followed by projection on $Y$. So $\left(\mathbf{P}^{3}\right)^{*} \times \Re_{d} \rightarrow$ $\mathfrak{K}_{d}$ is projective, and $\Sigma(F) \rightarrow\left(\mathbf{P}^{3}\right)^{*} \times \Re_{d}$ is projective since $F$ is. A slight simplification of Mather's proof shows that the set of $M$ such that $f_{M}$ is locally stable at each point $\Gamma$ is a Zariski open subset of $\mathfrak{T}_{d}$. q.e.d.

Now $f: \Gamma \rightarrow\left(\mathbf{P}^{3}\right)^{*}$ is locally stable at each point of $\Gamma$ if and only if the germ of $f$ at each point of $\Gamma$ has one of the following four normal forms. In other words, for each $x \in \Gamma$ there exist analytic coordinate charts $\varphi:\left(C^{4}, 0\right) \rightarrow(\Gamma, x)$ and $\Psi:\left(\mathbf{C}^{3}, 0\right) \rightarrow\left(\left(\mathbf{P}^{3}\right)^{*}, f(x)\right)$ such that the germ at 0 of $\varphi^{-1} \circ f \circ \varphi$ is one of the following:

$$
\begin{aligned}
\left(x_{1}, x_{2}, x_{3}, x_{4}\right) \rightarrow & \text { (1) }\left(x_{2}, x_{3}, x_{4}\right), \\
& \text { (2) }\left(x_{1}^{2}+x_{2}^{2}, x_{3}, x_{4}\right), \\
& \text { (3) }\left(x_{1}^{2}+x_{2}^{3}+x_{2} x_{3}, x_{3}, x_{4}\right), \\
& \text { (4) }\left(x_{1}^{2}+x_{2}^{4}+x_{2}^{2} x_{3}+x_{2} x_{4}, x_{3}, x_{4}\right) .
\end{aligned}
$$

(1) is a nonsingular (submersive) germ of $f,(2)$ is a nondegenerate singularity of $f$, (3) corresponds to a point on the cuspidal edge of the critical locus of $f$, and (4) corresponds to a swallowtail point of the critical locus of $f$.

The classification of the germs of $f$ follows from Mather's theorem that $f$ is locally (infinitesimally) stable at each point of $\Gamma$ if and only if $f$ is transverse to the family of contact classes in the jet space $J^{k}\left(\Gamma,\left(\mathbf{P}^{3}\right)^{*}\right)$ for $k$ sufficiently large ( $k \geqslant 3$ is large enough). The contact classes in $J^{k}\left(\mathbf{C}^{4}, \mathbf{C}^{3}\right)$ of codimension $\leqslant 7$ and rank 2 correspond to unfoldings of contact classes in $J^{k}\left(\mathbf{C}^{2}, \mathbf{C}\right)$ of codimension $\leqslant 4$ and rank 0 . These classes are the types $A_{1}$ (fold), $A_{2}$ (cusp), and $A_{3}$ (swallowtail), which have the normal forms (3.4) (cf. [18, XVII, §6], [5]).

Remark. Mather's theorem states that $f$ is locally stable at each finite subset of $\Gamma$ if and only if $f$ is multitransverse to the family of contact classes in $J^{k}\left(\Gamma,\left(\mathbf{P}^{3}\right)^{*}\right)$. Multitransversality implies that the image singularities of $f$ are mutually transverse. So we obtain as a corollary that the set of surfaces in $\mathbf{P}^{3}$ whose dual varieties have the classical singularities (double point curves, triple points, cuspidal edges, cuspidal double points, swallowtail points) is a Zariski open subset of $\Re_{d}$ (cf. [20, p. 229], where this description is assumed).

To complete the proof of the openness part of Theorem (3.1), we need to check that the germ of $f$ has one of the forms (3.4) if and only if the Gauss map 
$\gamma$ has properties (1.7)(i) and (ii). This is true because $\gamma$ is the restriction of $f$ to its singular locus $\Sigma(f)$.

More precisely, if we assume that the germ of $f$ has a normal form (3.4), then properties (1.7) for $\gamma$ can be checked. First of all, the singular locus $\Pi$ of $\gamma$ is the set of points at which the germ of $f$ has the form (3) or (4) (cuspidal edge swallowtail of the dual surface), and this locus is indeed a smooth curve. We need more for property (1.7)(i): zero should be a regular value of the curvature $K$. This is equivalent to the statement that $d \gamma: T(M) \rightarrow T\left(\left(\mathbf{P}^{3}\right)^{*}\right)$ defines a section of the bundle $\operatorname{HOM}\left(T(M),\left.T\left(\left(\mathbf{P}^{3}\right)^{*}\right)\right|_{M}\right)$ which is transverse to the subbundle of homomorphisms of rank 1. Now $\left.T\left(\left(\mathbf{P}^{3}\right)^{*}\right)\right|_{M}=$ $\operatorname{HOM}(\tilde{T}(M), N(M))$, and $d \gamma$ factors to induce a map $T(M) \rightarrow$ $\operatorname{HOM}(T(M), N(M))$ (cf. [12, p. 379]), which can be identified with the restriction of $d f$ to $T(\Sigma(f)) \rightarrow$ Image $(d f)$. (Note that $d f$ has rank 2 on $\Sigma(f)$.) It is easy to check that for $f$ of the form (3) or (4), this restriction defines a section of the bundle $\operatorname{HOM}(T(\Sigma(f))$, Image $(d f))$ which is transverse to the subbundle of homomorphisms of rank 1.

To check property (1.7)(ii) for the germs (3) and (4), note that the kernel line of $d \gamma$ becomes tangent to the singular locus of $\gamma$ precisely at points where the germ of $f$ has type (4) (a swallowtail of the dual surface). It is easy to check using the normal form (4) that this tangency is nondegenerate. In fact, $\gamma=f \mid \Sigma(f)$ has rank 1 along the smooth curve $\Pi=\Sigma(f \mid(\Sigma(f)))$, and the tangency is nondegenerate because 0 is a regular value of the restriction of $d \gamma$ to $T(\Pi) \rightarrow$ Image $(d \gamma)$. The normal form (4) also shows that the curve $\gamma(\Pi)$ in $\left(\mathbf{P}^{3}\right)^{*}$ has a cusp wherever $\operatorname{ker}(d \gamma)$ is tangent to $\Pi$. This is why we call these points "cusps" of the Gauss map (although "swallowtails" might be more appropriate).

Now suppose that $\gamma=f \mid \Sigma(f)$ has properties (1.7)(i), (ii). The jet extension of $f$ is automatically transverse to the contact class $A_{1}$. A standard argument shows that it is transverse to $A_{2}$ if and only if (i) holds, and transverse to $A_{3}$ if and only if (ii) holds (cf. [10, Chapter VII, §3]).

To complete the proof of the theorem, we must show that for each $d \geqslant 2$ there exists a surface with properties (1.7)(i)(ii). Rather than trying to construct such surfaces, we appeal to a topological transversality argument of Bruce [8]. His result implies that (1.7) holds for all $M$ in the complement of a real subanalytic subset of real codimension at least one in $\Re_{d}$. The basic idea is to think of the family of hyperplane sections of $M$ locally as a family of "height functions" on $M$, and to show that by perturbing $M$ this family can be made transverse to strata of the space of $k$-jets of functions on $M$ [8, Theorem 2.4]. Some choices are necessary to define the family of height functions locally on 
$M$, but Bruce shows that different choices give equivalent germs of families [8, Lemma 2.1]. To apply his theorem to the Gauss map, we must explain the relation between his construction and the map $f_{M}$ (3.2). Since his construction is local, we can replace $M$ by an affine neighborhood $N$ of $P$, say $N \subset \mathrm{C}^{3}$ with coordinates $(x, y, z), P=0$, and the plane $z=0$ tangent to $N$ at $P$. Choose a linear map $L: \mathbf{C}^{2} \rightarrow\left(\mathbf{C}^{3}\right)^{*}$ so that the image of $L$ does not contain the coordinate function $z$. Then Bruce defines a germ of a family of functions $H$ $\left(N \times \mathbf{C}^{2}, 0\right) \rightarrow(\mathbf{C}, 0)$ by $H(u, v)=(z+L(v))(u)$. Define

$$
H^{\prime}:\left(N \times \mathrm{C}^{2}, 0\right) \rightarrow\left(\mathrm{C} \times \mathrm{C}^{2}, 0\right), \quad H^{*}(u, v)=(H(u, v), v) .
$$

It is easy to see that the map germ (3.5) is equivalent to the germ of $f_{M}(3.2)$ at $\left(x, \tilde{T}_{x} M\right)$.

An immediate corollary of Bruce's transversality theorem [8, Theorem 2.4] is that for $M \in \Re_{d}-B, B$ a proper real subanalytic set of $\Re_{d}$, the family germ $H$ is transverse to the singularities $A_{1}, A_{2}$, and $A_{3}$ in $J^{4}\left(\mathbf{C}^{2}, \mathbf{C}\right)$, and misses the other strata of the jet space. (A special argument is needed for $d=3[8, \mathrm{pp}$. 52-53].) It follows that the map germ $f_{M} \sim H^{\prime}$ must be equivalent to $(3.4)(1)(2)(3)$ or (4). This completes the proof of Theorem (3.1).

Remark. Bruce proves a corresponding multitransversality theorem only for surfaces of sufficiently large degree $(d \geqslant 9)$.

For real surfaces there is a similar result:

(3.6) Theorem. Let $M$ be a compact $C^{\infty}$ surface. The set of embeddings of $M$ in $\mathbf{R} P^{3}$ with properties (1.7)(i), (ii) is an open dense subset of $C^{\infty}\left(M, \mathbf{R} P^{3}\right)$ (Whitney topology).

Proof. Bruce's construction of the germ $H$ of a family of height functions works equally well in this case, as well as his proof that the germ of $H$ is independent of the choices involved. (It is not necessary to have $M$ algebraic.) But for a surface in $\mathbf{R}^{3}$, the germ $H$ at $x \in M$ is equivalent to the germ of the family of orthogonal projections to oriented lines in $\mathbf{R}^{3}$ at the point $(x, l), l$ the normal line to $M$ at $x$. Bruce $[8, \S 1]$ shows, using results of Looijenga, that the set of embeddings of a smooth surface $N$ in $\mathbf{R}^{3}$ for which the family of orthogonal projections to lines is transverse to a stratified subset of $J^{k}\left(\mathbf{R}^{2}, \mathbf{R}\right)$ is open and dense in $C^{\infty}\left(N, \mathbf{R}^{3}\right)$. The theorem follows by the compactness of $M$.

The result for surfaces in $\mathbf{R}^{3}$ also follows from the work of Bleeker and Wilson [7]. They give an elementary proof that property (1.7)(i), (ii) is generic for surfaces in $\mathbf{R}^{3}$ by direct analysis of the classical Gauss map $M \rightarrow S^{2}$.

Remark. Another approach to the singularities of the Gauss map is via Arnold's theory of Legendre singularities [1], [5]. The Gauss map $M \rightarrow\left(\mathbf{P}^{3}\right)^{*}$ is a Legendre mapping, and so it can be expected to have Legendre singularities-cuspidal edges and swallowtails. 


\section{References}

[1] V. I. Arnold, Critical points of smooth functions, Proc. Internat. Congr. Math., Vancouver, 1974, 19-39.

[2] __ Geometrical methods in the theory of ordinary differential equations, Springer, New York, 1983.

[3]___ Indices of singular points of 1-forms on a manifold with boundary, convolution of invariants of reflection groups, and singularities of projections of smooth surfaces, Uspehi Mat. Nauk 34:2 (1979) 3-38; English transl. in Russian Math. Surveys 34:2 (1979) 1-42.

[4] Singularities in systems of rays, Uspehi Mat. Nauk 38:2 (1983) 77-147.

[5] V. I. Arnold, A. N. Varchenko \& S. M. Gusein-Zade, Singularities of differentiable mappings, "Nauka", Moscow 1981; English transl., Birkhäuser, Boston, to appear.

[6] T. Banchoff, T. Gaffney \& C. McCrory, Cusps of Gauss mappings, Research Notes in Math., No. 55, Pitman, Boston, 1982.

[7] D. Bleeker \& L. Wilson, Stability of Gauss maps, Illinois J. Math. 22 (1978) 279-289.

[8] J. W. Bruce, The duals of generic hypersurfaces, Math. Scand. 49 (1981) 36-60.

[9] E. Cartan, Leçons sur la géométrie projective complexe, Gauthiers-Villars, Paris, 1931.

[10] M. Golubitsky \& V. Guillemin, Stable mappings and their singularities, Springer, New York, 1973.

[11] P. Griffiths \& J. Harris, Principles of algebraic geometry, Wiley, New York, 1978.

[12] Algebraic geometry and local differential geometry, Ann. Sci. École Norm. Sup. (4) 12 (1979) 355-452.

[13] Y. L. Kergosien \& R. Thom, Sur les points paraboliques des surfaces, C. R. Acad. Sci. Paris Sér. A-B, 299 (1980) 705-710.

[14] J. King, The currents defined by analytic varieties, Acta Math. 127 (1971) 185-220.

[15] F. Klein, Über Flächen dritter Ordunung, Gesammelte Mathematische Abhandlungen, II Band, Berlin, 1922, 11-62.

[16] V. S. Kulikov, Calculation of singularities of embeddings of generic algebraic surfaces in projective space $\mathbf{P}^{3}$, Funktsional. Anal. i Prilozhen. 17:3 (1983) 15-27.

[16a] On the number of special local centers of projection of an algebraic surface, Uspehi Mat. Nauk 38 (1983).

[17] E. E. Landis, Tangential singularities, Funktsional. Anal. i Prilozhen. 15:2 (1981) 36-49; English transl. in Functional Anal. Appl. 15 (1981) 103-114.

[18] J. Martinet, Singularities of smooth functions and maps, London Math. Soc. Lecture Notes No. 58, Cambridge, 1982.

[19] J. Mather, Stable map-germs and algebraic geometry, Manifolds-Amsterdam, Lecture Notes in Math., Vol. 197, Springer, Berlin and New York, 1970, 176-193.

[20] R. Piene, Some formulas for a surface in $\mathbf{P}^{3}$, Algebraic Geometry, Lecture Notes in Math., Vol. 678, Springer, Berlin and New York, 1978, 196-235.

[21] O. A. Platonova, Singularities of contact of a surface and a line, Uspehi Mat. Nauk 36:1 (1981) 221-222; Russian Math. Surveys 36:1 (1981) 248-249.

[22] G. Salmon, $A$ treatise on the analytic geometry of three dimensions, 4th ed., Dublin, 1882.

[23] T. Shifrin, The kinematic formula in complex integral geometry, Trans. Amer. Math. Soc. 264 (1981) 255-293.

[24] M. Spivak, A comprehensive introduction to differential geometry, Vol. III, Publish or Perish, Boston, 1979.

UNIVERSITY OF GEORGIA 